\title{
Curcumin Inhibits Proliferation and Epithelial-Mesenchymal Transition in Lens Epithelial Cells through Multiple Pathways
}

\author{
Huijun Liu, ${ }^{1}$ Yuxiang Mao, ${ }^{1}$ Bing Xia, ${ }^{2}$ Chongde Long, ${ }^{1}$ Xielan Kuang, ${ }^{1,3}$ Hao Huang, \\ Jie Ning, ${ }^{1}$ Xinqi Ma, ${ }^{1}$ Han Zhang, ${ }^{1}$ Renchun Wang, ${ }^{4}$ Han Tang, ${ }^{1}$ Han Du, ${ }^{1}$ Jianhua Yan $\left(\mathbb{D},{ }^{1}\right.$ \\ Qingjiong Zhang, ${ }^{1}$ Xinyu Zhang $\mathbb{D}^{1}$, and Huangxuan Shen $\mathbb{D}^{1,3}$
}

\author{
${ }^{1}$ State Key Laboratory of Ophthalmology, Zhongshan Ophthalmic Center, Sun Yat-sen University, Guangzhou 510060, China \\ ${ }^{2}$ Hunan Cancer Hospital and the Affiliated Tumor Hospital of Xiang-Ya School of Medicine, Central South University, \\ Changsha 410078, China \\ ${ }^{3}$ Biobank of Eye, State Key Laboratory of Ophthalmology, Zhongshan Ophthalmic Center, Sun Yat-sen University, \\ Guangzhou 510060, China \\ ${ }^{4}$ The Second Clinical Medicine School of Lanzhou University, Lanzhou 730000, China
}

Correspondence should be addressed to Xinyu Zhang; zhangxy7@mail.sysu.edu.cn and Huangxuan Shen; shenhx@mail.sysu.edu.cn

Received 8 January 2020; Revised 23 February 2020; Accepted 4 March 2020; Published 2 April 2020

Academic Editor: Diego Franco

Copyright (c) 2020 Huijun Liu et al. This is an open access article distributed under the Creative Commons Attribution License, which permits unrestricted use, distribution, and reproduction in any medium, provided the original work is properly cited.

\begin{abstract}
Background. Posterior capsule opacification (PCO), a complication of extracapsular lens extraction surgery that causes visual impairment, is characterized by aberrant proliferation and epithelial-mesenchymal transition (EMT) of lens epithelial cells (LECs). Curcumin, exerting inhibitive effects on cell proliferation and EMT in cancer, serves as a possible antidote towards PCO. Methods. Cellular proliferation of LECs after treatment of curcumin was measured with MTT assay and flow cytometry. The transcriptional and expressional levels of proteins related to proliferation and EMT of LECs were quantified by western blotting and real-time PCR. Results. Curcumin was found to suppress the proliferation of LECs by inducing $\mathrm{G}_{2} / \mathrm{M}$ arrest via possible inhibition of cell cycle-related proteins including CDK1, cyclin B1, and CDC25C. It had also inactivated proliferation pathways involving ERK1/2 and Akt pathways in LECs. On the other hand, curcumin downregulated the EMT of LECs through blocking the TGF- $\beta /$ Smad pathway and interfering Notch pathway which play important roles in PCO. Conclusions. This study shows that curcumin could suppress the proliferation and EMT in LECs, and it might be a potential therapeutic protection against visual loss induced by PCO.
\end{abstract}

\section{Introduction}

Cataract is a vision-impairing disease in which opacification and turbidness of the lens are formed. Various factors contribute to the pathological process of cataract, while the only solution is performing ophthalmic surgery extracting a muddy lens out of the capsule with or without the intraocular lens implantation. However, these surgeries are risky with complications. Posterior capsule opacification (PCO) is a common complication after cataract surgeries and causes setback of sight during postoperation period [1]. PCO occurs in $30 \%$ of adults undertaking the modern lens surgery [2], and
$100 \%$ in children owning to their strong proliferation ability of lens epithelial cells (LECs). Additional treatments including lens capsule polishing, laser capsulotomy, posterior continuous curvilinear capsulorhexis, and anterior vitrectomy are only able to decrease the incidence by $15 \%$, and they might increase the risk of postsurgical inflammation and other complications [3,4]. Hirnschall showed a method of opening capsular bag that significantly reduced PCO in rabbits, but procedures in human eyes were not carried out [2]. A new surgical method of cataract removal in infants was reported lately, which had preserved endogenous LECs and achieved functional lens regeneration, avoiding the 
complication of PCO [5]. But for cataract in elder children and adults, effective prevention and therapy of PCO are still in huge demand.

Epithelial-mesenchymal transition EMT, a main characteristic process of PCO, includes the acquisition of mesenchymal cell-like morphology, diminished intercellular junctions, reduced expression of epithelial markers such as ZO-1 and E-cadherin, and accumulation of EMT markers including $\alpha$-smooth muscle actin ( $\alpha$-SMA), fibronectin, and $\mathrm{N}$-cadherin, in addition to the upregulated expression of transcription factors including Snail, Slug, ZEB, and Twist $[1,4]$. Among different EMT inducers, transforming growth factor beta (TGF- $\beta$ ) is the best known. The TGF- $\beta$ family causes biological reactions by mediating Smad transcription factors and regulates gene's expression. It also activates non-Smad pathways that exert nontranscriptional roles including epithelial junction dissolution, cytoskeletal reorganization, motility, and translational control. There are three isoforms of TGF- $\beta$, and they are all identified in humans. Among them, TGF- $\beta 2$ is the predominant isoform in ocular media and the most important factor contributing to transdifferentiation and pathologic fibrosis of LECs $[1,4,6,7]$.

Curcumin as a component of turmeric (Curcuma longa), 6-heptadiene-3,5-dione,1,7-bis(4-hydroxy-3-methoxyphenyl)-1,(e,e)-1 with the CAS number: 458-37-7, has been medically used in Asian for centuries [8]. Curcumin is a natural polyphenol and recent researches have revealed its diverse biological functions including antioxidation, antifungus, antibacteria, antivirus, anti-inflammation, immune-modulation, antiproliferation, and tumor suppression [8-12]. Besides, curcumin plays a regulatory role in the metabolic diseases through hypoglycemic, lipid-lowering, and cardioprotection mechanisms [13-15]. Despite the universal functions, studies of curcumin were mostly focused on the antitumor effect in cancer therapy. One of the vital mechanisms of this effect was found to be inhibiting proliferation and EMT in cancers, where pleiotropic genetic effects and multiple cell signaling pathways participated [16-18]. Thus, it is highly possible that curcumin exhibits proliferation and EMT blocking effects on LECs, which leads to a potential solution of PCO.

In this study, we confirmed the abilities of curcumin inhibiting proliferation and TGF- $\beta 2$-induced EMT of human LECs according to a dose-dependent manner involving multiple pathways. In addition to its various effects, curcumin was shown in our investigation to become a novel cure of cataract surgical PCO.

\section{Materials and Methods}

The study was conducted in accordance with the Basic and Clinical Pharmacology and Toxicology policy for experimental and clinical studies [19].

2.1. Cell Culture and Treatments. The LEC cell line SRA01/04 was cultured in Dulbecco's modified Eagle's medium (DMEM, Life Technologies, Auckland, New Zealand) containing 10\% fetal bovine serum (Life Technologies, Auckland, New Zealand). Cells were grown in a humidified atmosphere containing $5 \% \mathrm{CO}_{2}$ at $37^{\circ} \mathrm{C}$ and dissociated with $0.25 \%$ trypsin- $0.04 \%$ EDTA solution. TGF- $\beta 2$ was reconstituted with $4 \mathrm{mM} \mathrm{HCl}$ containing $0.1 \%$ BSA to a final concentration of $50 \mu \mathrm{g} / \mathrm{ml}$, and curcumin was dissolved in DMSO to a storage concentration of $1 \mathrm{mM}$. For the treatments with TGF- $\beta 2$ or curcumin, cells were first seeded in $100 \mathrm{~mm}$ dishes, then treated with $10 \mathrm{ng} / \mathrm{ml}$ recombinant human TGF- $\beta 2$ or various concentrations of curcumin for $48 \mathrm{~h}$.

2.2. Cell Proliferation Assay. The proliferation of LECs under curcumin treatment was assessed with MTT assay. LECs were seeded into 96 -well plates at a density of $4 \times 10^{3}$ cells/well and grown overnight. Then, cells were treated with different doses $(0 \mu \mathrm{M}, 5 \mu \mathrm{M}, 10 \mu \mathrm{M}, 20 \mu \mathrm{M}$, and $40 \mu \mathrm{M})$ of curcumin (Sigma-Aldrich, Louis, MO, USA) for $24 \mathrm{~h}, 48 \mathrm{~h}$, and $72 \mathrm{~h}$, respectively. The following procedures were conducted as described previously [20].

2.3. Cell Apoptosis and Cell Cycle Analysis. LECs treated with different concentrations of curcumin for $48 \mathrm{~h}$ were collected and washed with PBS; cell apoptosis and cell cycle were analyzed by flow cytometry (Millipore, Darmstadt, Hesse, Germany) with the MultiCaspase Kit and the Cell Cycle Kit (Millipore, Darmstadt, Hesse, Germany). All procedures were proceeded according to the manufacturer's protocols as we previously did $[21,22]$. The statistical analysis was performed with the software version 1.3 of the Muse ${ }^{\mathrm{TM}}$ Cell Analyzer (Millipore, Darmstadt, Hesse, Germany).

2.4. Real-Time RT-PCR. Total RNA of cells treated with TGF- $\beta 2$ or curcumin for $48 \mathrm{~h}$ was isolated with TRIzol reagent (Takara Bio, Kusatsu, Shiga, Japan). cDNA synthesis and real-time RT-PCR were underwent as previously described [20, 23]. Data collection and analysis were used with the additional application software (LightCycler 96, F. Hoffmann-La Roche Ltd.) version 1.1. And the relative quantitative analysis of mRNA was calculated by using the $2^{-\Delta \Delta \mathrm{Cq}}$ method with normalization to GAPDH.

2.5. Western Blot Assay. Cell proteins were extracted with a lysis buffer solution containing phosphatase inhibitor from the LECs. The western blot procedures were referred to our previous work [20, 23]. GAPDH (Proteintech, Wuhan, Hubei, China) and tubulin alpha (Affinity Biotech, Kansas, $\mathrm{MO}$, USA) were used as controls. Other antibodies used included CDK1, cyclin B1, CDC25C, E-cadherin, fibronectin, $\alpha$-SMA (Boster, Wuhan, Hubei, China), Notch1, Notch2, Smad2/3, p-Smad2/3 (Santa Cruz, CA, USA), p38, p-P38, Akt, p-Akt, ERK1/2, and p-ERK1/2 (CST, MA, USA).

2.6. Statistical Analysis. Each experiment was performed in triplicate with same protocol. All data were expressed as mean \pm S.E.M, and statistical analyses were processed with SPSS version 22.0 (SPSS Inc., Chicago, IL, USA). One-way analysis of variance was used to compare differences among groups. $P<0.05$ is regarded as statistically significant. 


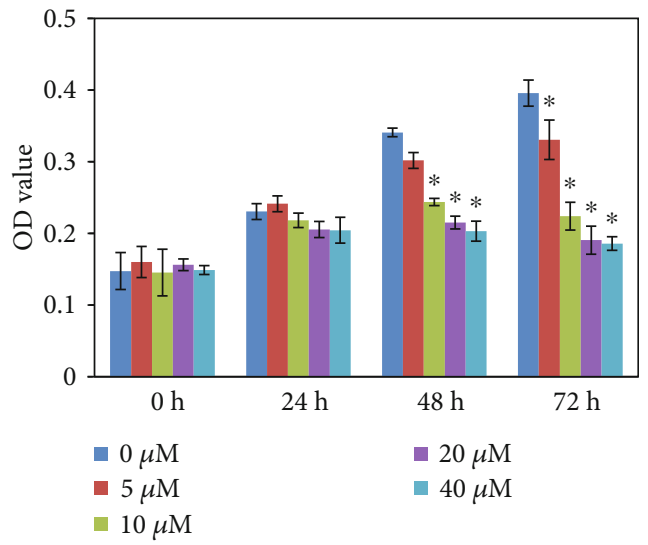

(a)

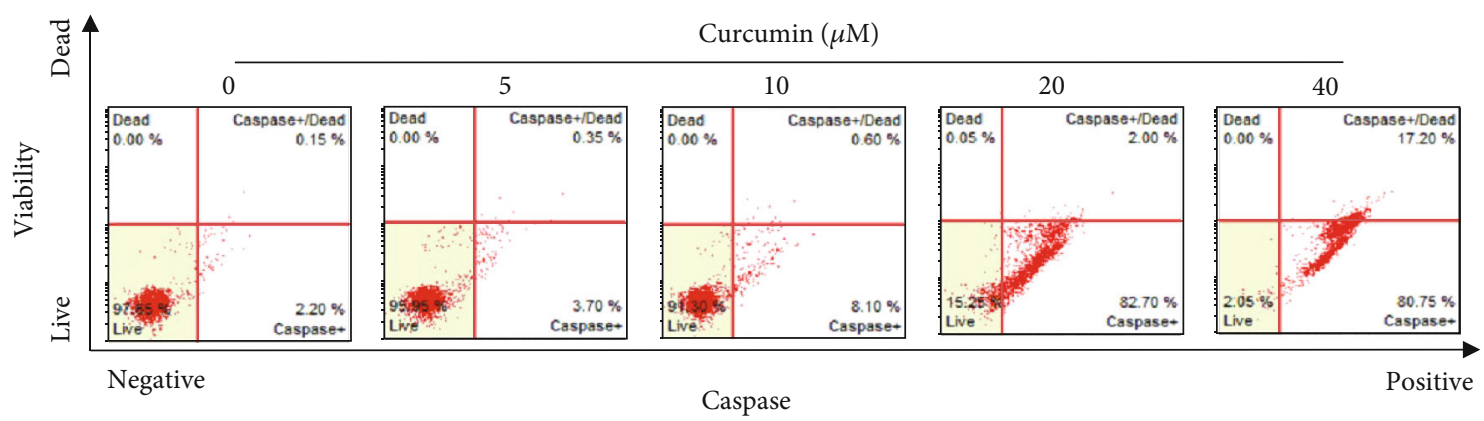

(b)

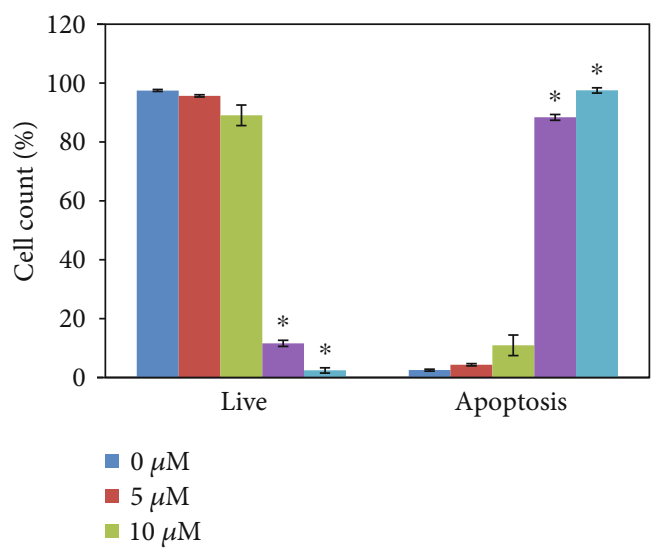

(c)

FIGURE 1: Curcumin treatments inhibited proliferation and increased apoptosis of LEC cells. (a) LECs were treated with curcumin $(0,5,10$, 20 , and $40 \mu \mathrm{M}$ ) for $72 \mathrm{~h}$, and cell viabilities were assessed with an MTT assay in different time points. (b) LEC apoptosis was measured by flow cytometry using the MultiCaspase Kit after treatment with curcumin $(0,5,10,20$, and $40 \mu \mathrm{M})$ for $48 \mathrm{~h}$. (c) A histogram of cell viability and cell apoptosis in (b) is shown. ${ }^{*} P<0.05$ vs. cells treated without curcumin.

\section{Results}

3.1. Curcumin Inhibited the Proliferation of LECs. To investigate whether curcumin can prevent the aberrant proliferation of LECs, we used gradient concentrations of curcumin on SRA01/04 for $72 \mathrm{~h}$. The MTT assay revealed that the proliferation of LECs was suppressed by curcumin in a time-dose-dependent manner (Figure 1(a)). Meanwhile, concentrations above $10 \mu \mathrm{M}$ had led to approximate OD values after the time point of $24 \mathrm{~h}$, which suggested that the LEC cell growth arrest might be induced by $10^{+} \mu \mathrm{M}$ of curcumin in vitro. On the other hand, curcumin had induced apoptosis of LECs. Flow cytometry showed that curcumin had raised cell apoptosis to $(88.4 \pm 6.09) \%$ and $(97.5 \pm 0.90) \%$ at the concentration of $20 \mu \mathrm{M}$ and $40 \mu \mathrm{M}$ after $48 \mathrm{~h}$, compared with the control group $(2.53 \pm 0.32) \%$ (Figures $1(\mathrm{~b})$ and $1(\mathrm{c})$ ). However, the dosages of curcumin below $10 \mu \mathrm{M}$ did not affect cell viability significantly $(P>0.05)$.

3.2. Curcumin Inhibited Cell Cycle Progression of LECs at $G_{2} / M$ Phase. Cell cycle was analyzed with flow cytometry after LECs were treated with curcumin for $48 \mathrm{~h}$. The results 


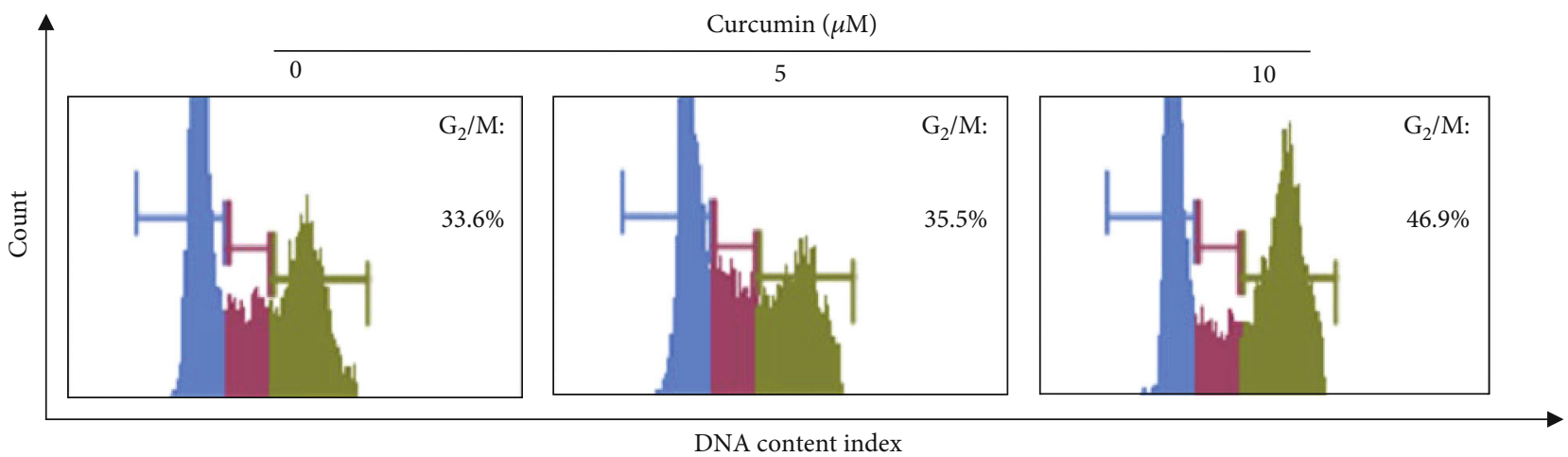

(a)

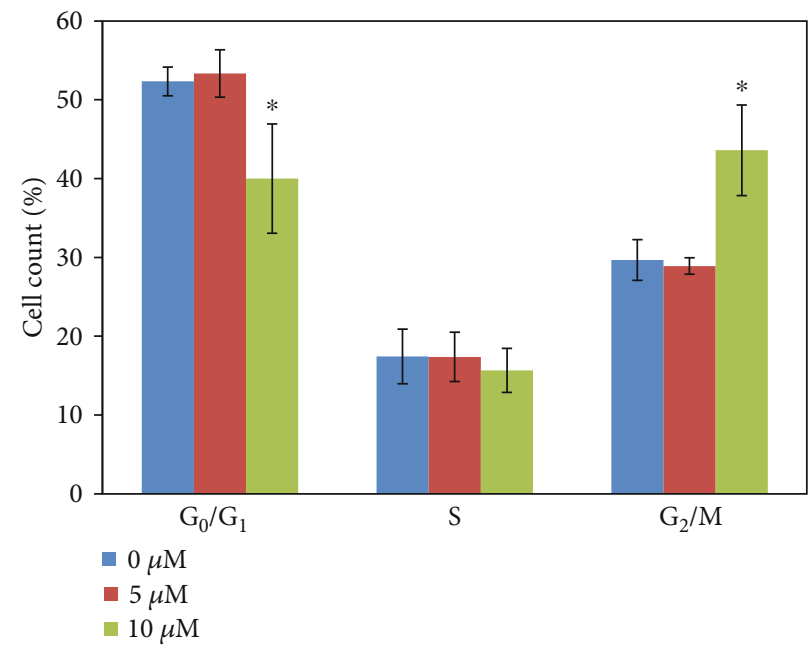

(b)

Figure 2: Curcumin induced cell cycle arrest in G2/M phase. (a) Cell cycle of LECs treated with curcumin $(0,5$, and $10 \mu \mathrm{M})$ were examined by flow cytometry. (b) A histogram of cell cycle in (a) is shown. ${ }^{*} P<0.05$ vs. cells treated without curcumin.

showed that the curcumin of $10 \mu \mathrm{M}$ had increased cell numbers in the $\mathrm{G}_{2} / \mathrm{M}$ phase $(43.6 \pm 5.75) \%$, while $5 \mu \mathrm{M}$ curcumin did not affect the $\mathrm{LEC}$ cycle in the $\mathrm{G}_{2} / \mathrm{M}$ phase $(28.9 \pm 1.04) \%$ $(P>0.05)$, compared with the control $(29.7 \pm 2.58) \%$ $(P<0.05)$ (Figure 2). The cell cycle-regulating proteins of the $\mathrm{G}_{2} / \mathrm{M}$ phase were also examined. At the dose of $10 \mu \mathrm{M}$, for $48 \mathrm{~h}$, treatment of curcumin had downregulated the RNA transcriptional levels of CDK1 and cyclin B1. Meanwhile, proteins including CDK1, cyclin B1, and CDC25C were inhibited significantly (Figure 3 ). But interestingly, curcumin of $5 \mu \mathrm{M}$ increased the protein expression of CDK1.

3.3. Curcumin Suppressed TGF- $\beta 2$-Induced EMT of LECs. We had examined the RNA transcriptional levels of recombinant human TGF- $\beta 2$-induced EMT level in LECs at the concentration of $10 \mathrm{ng} / \mathrm{ml}$ for $48 \mathrm{~h}$. Firstly, gene expression markers of EMT including fibronectin, collagen I, collagen $I V$, and $N$-cadherin, and transcription factor Snail were all elevated correspondingly (Figure 4(a)). Secondly, in the LECs cocultured with curcumin of different concentrations $(0 \mu \mathrm{M}$, $5 \mu \mathrm{M}$, and $10 \mu \mathrm{M})$ and TGF- $\beta 2$ of $10 \mathrm{ng} / \mathrm{ml}$, the transcriptional levels of fibronectin, collagen $I$, and collagen $I V$ were decreased in a dose-dependent manner (Figure 4(b)). By contrast, WB showed that the protein expressions of fibronectin and $\alpha$-SMA were decreased, while E-cadherin was increased under cotreatment of curcumin and TGF- $\beta 2$ (Figures 5(a) and $5(\mathrm{~b}))$. Last but not the least, curcumin had inhibited the TGF- $\beta 2$-induced phosphorylation of $\operatorname{Smad} 2 / 3$ and hence suppressed the TGF- $\beta 2$ signaling pathway (Figure 5).

3.4. Curcumin and TGF- $\beta 2$ Interacted with the Notch Signaling Pathway. The transcriptional level of Notch1 and Notch2 RNAs elevated in LECs treated with curcumin, TGF- $\beta 2$, or both. However, the expressions of Notch were shown to be reducing in LECs with cotreatment of TGF- $\beta 2$ and curcumin (Figure 6).

3.5. Curcumin Blocked the ERK1/2 and Akt Signaling Pathway. Both the ERK1/2 pathway and Akt signaling pathway are connected with proliferation and cell growth [4]. WB showed that curcumin had decreased phosphorylation of ERK1/2 and Akt induced by TGF- $\beta 2$. In this way, curcumin was capable of blocking the ERK1/2 and Akt signaling pathways; thus, it is able to regulate cell proliferation and growth. However, p38 and p-P38 of the MAPK pathway were not changed during TGF- $\beta 2$ and curcumin treatments (Figures $7(\mathrm{a})$ and $7(\mathrm{~b})$ ). 

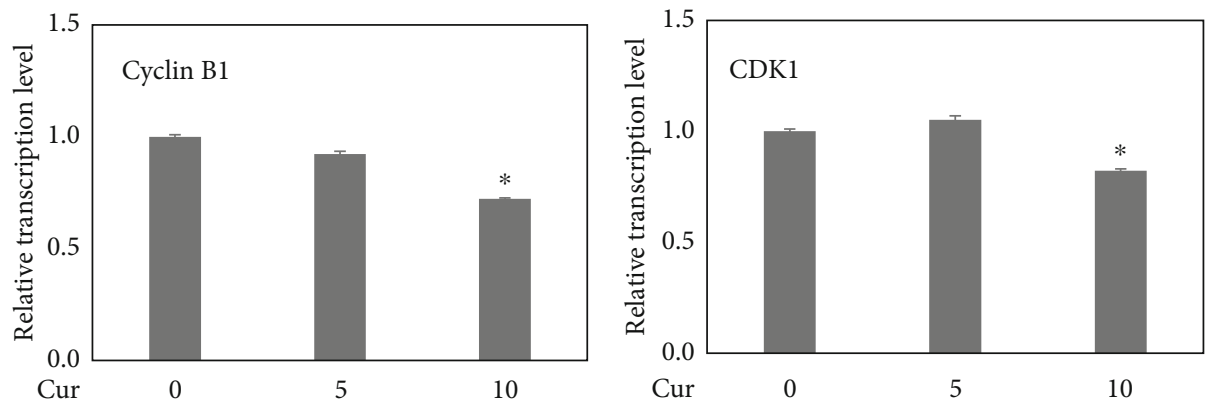

(a)
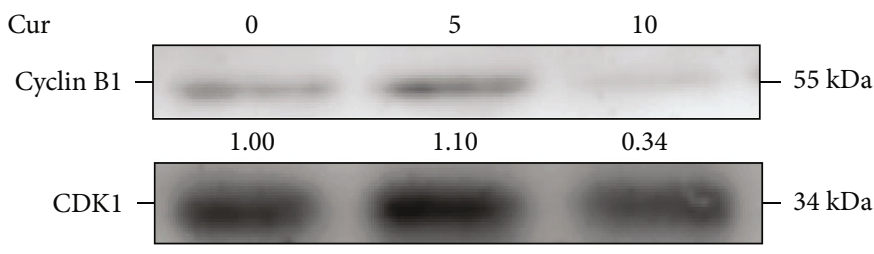

$\begin{array}{lll}1.00 & 1.37 & 1.01\end{array}$
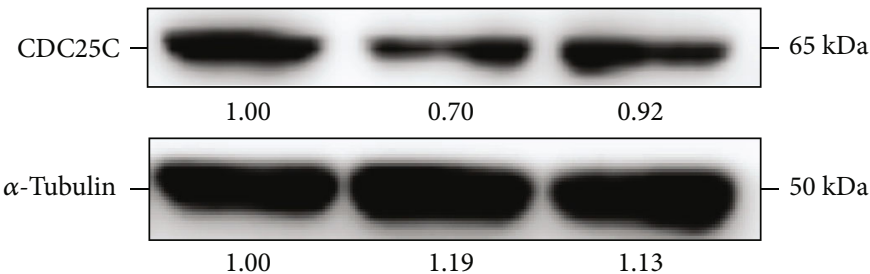

(b)
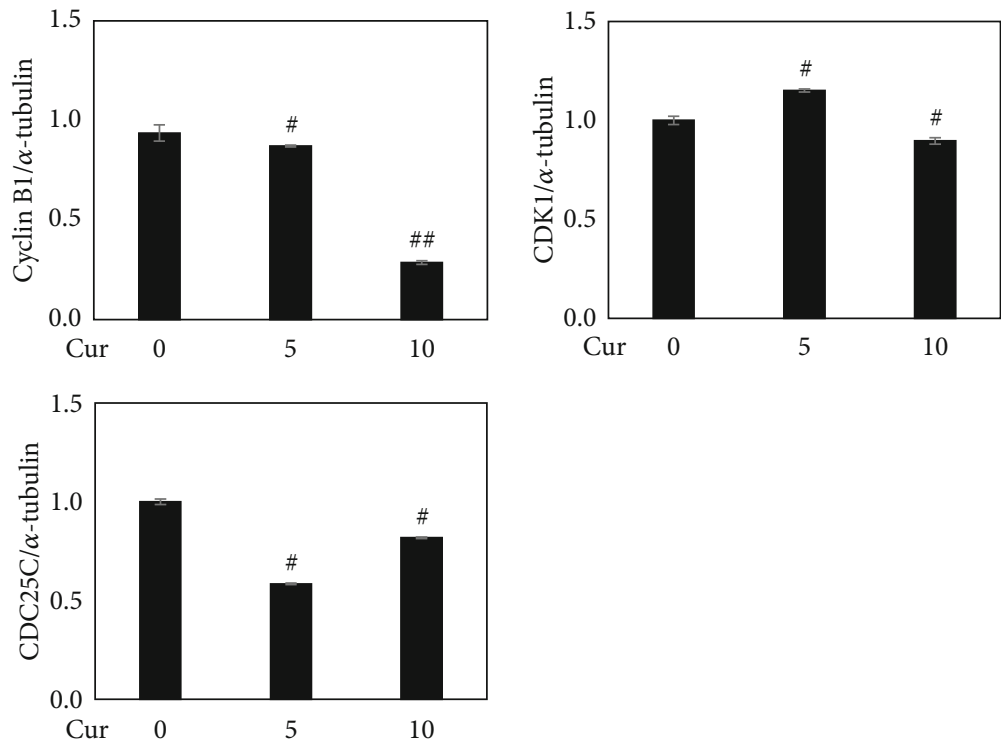

(c)

FIgURE 3: Curcumin decreased mRNA transcription and protein expression of cell cycle-related proteins at $10 \mu \mathrm{M}$. (a) The mRNA transcriptions of cell cycle-related proteins cyclin B1 and CDK1 in LECs treated with curcumin $(0,5$, and $10 \mu \mathrm{M})$ for $48 \mathrm{~h}$ were detected by real-time PCR. GAPDH was used as an internal control. ${ }^{*} P<0.05$, vs. the control. (b) Expressions of cell cycle-related proteins cyclin $\mathrm{B} 1, \mathrm{CDK} 1$, and CDC25C in curcumin-treated LECs were determined using western blot analysis. $\alpha$-Tubulin was used as an internal control. (c) Densitometric analyses of the protein expression levels of cyclin B1, CDK1, and CDC25C from the western blots are shown. ${ }^{\#} P<0.05,{ }^{\#} P<0.01$ vs. the control.

\section{Discussion}

This study has revealed the potential value of curcumin in PCO treatment for the first time. PCO is one of the most common complications after cataract surgery, which manifests visual loss resulting from proliferation and EMT of LECs. Despite posterior capsulotomy by using laser or surgery being accessible for PCO treatment, they will increase 

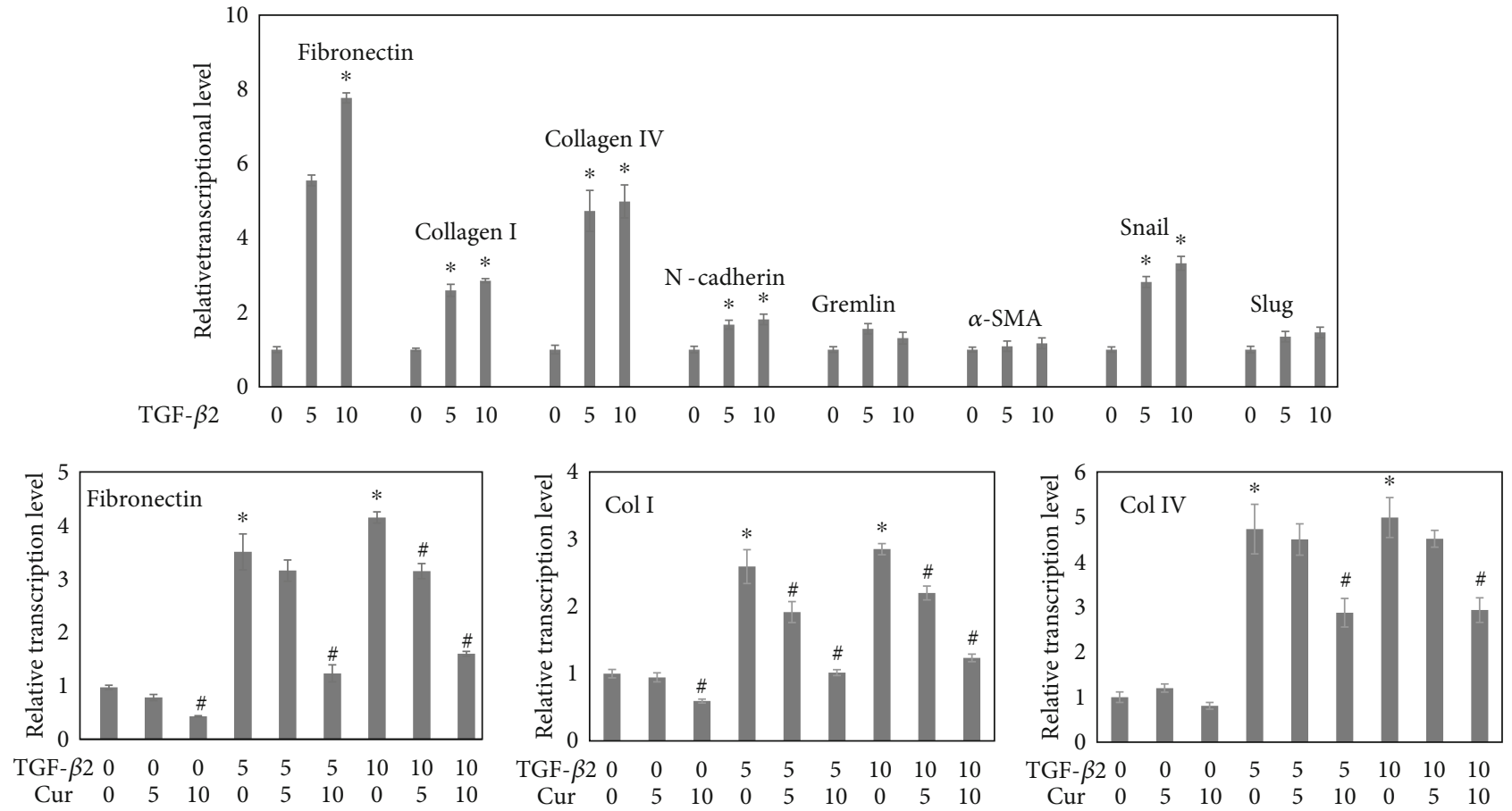

FIGURE 4: Curcumin inhibited the transcription of EMT-related genes induced by TGF- $\beta 2$ in a dose-dependent manner. (a) Normalized mRNA levels of EMT-related genes (fibronectin, collagen I, collagen IV, etc.) in LECs treated with TGF- $\beta 2$ ( 0 , 5 , and $10 \mathrm{ng} / \mathrm{ml})$ for $24 \mathrm{~h}$ were detected by real-time PCR. (b) Normalized mRNA levels of EMT-related genes (fibronectin, collagen I, and collagen IV) in LECs treated with both TGF- $\beta 2(0,5$, and $10 \mathrm{ng} / \mathrm{ml})$ and curcumin $(0,5$, and $10 \mu \mathrm{M})$ for $24 \mathrm{~h}$ were detected by real-time PCR. ${ }^{*} P<0.05$ vs. the control, ${ }^{\#} P<0.05$ vs. cells without curcumin pretreatment.

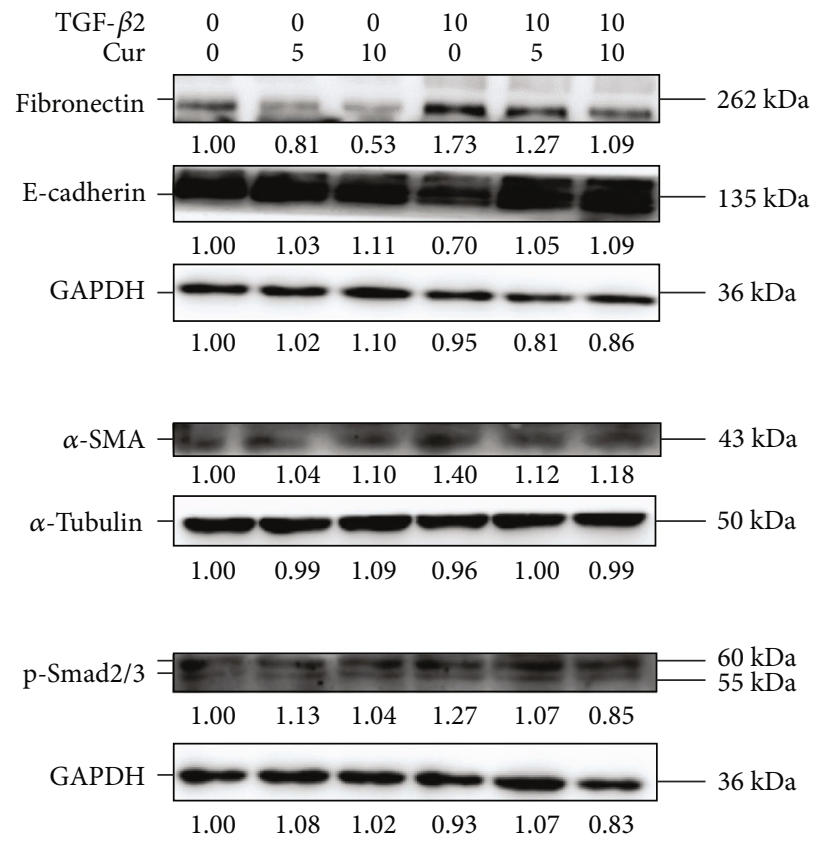

(a)
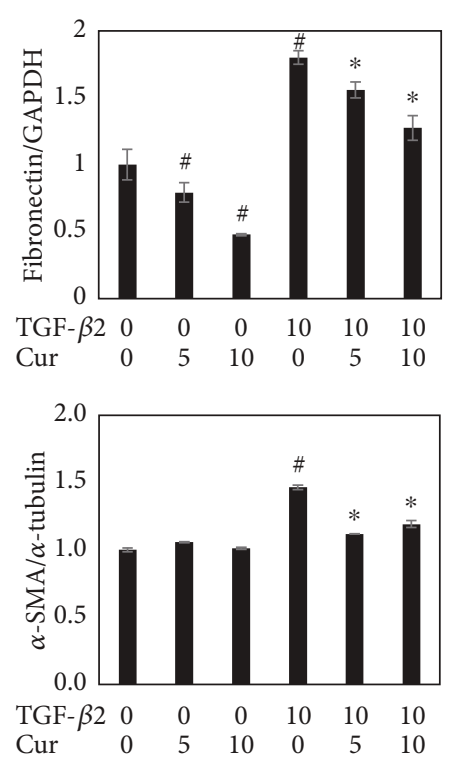

(b)
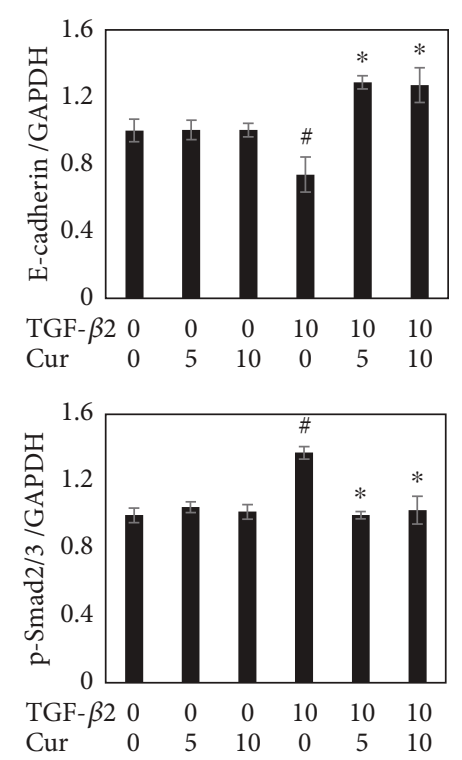

FIGURE 5: Curcumin suppressed the TGF- $\beta 2$-induced EMT and TGF- $\beta /$ Smad pathway activation. (a) EMT-related protein (fibronectin, $\alpha$-SMA, and E-cadherin) and phosphorylated Smad $2 / 3$ in cells treated with TGF- $\beta 2(0,10 \mathrm{ng} / \mathrm{ml})$ and curcumin $(0,5$, and $10 \mu \mathrm{M})$ for $24 \mathrm{~h}$ were detected by western blots. (b) Densitometric analyses of the protein expression levels of fibronectin, E-cadherin, $\alpha$-SMA, and p-Smad2/3 from the western blots are shown. GAPDH and $\alpha$-tubulin were used as internal controls. ${ }^{\#} P<0.05$ vs. the control, ${ }^{*} P<0.05$ vs. treatment with TGF- $\beta 2$ only. 

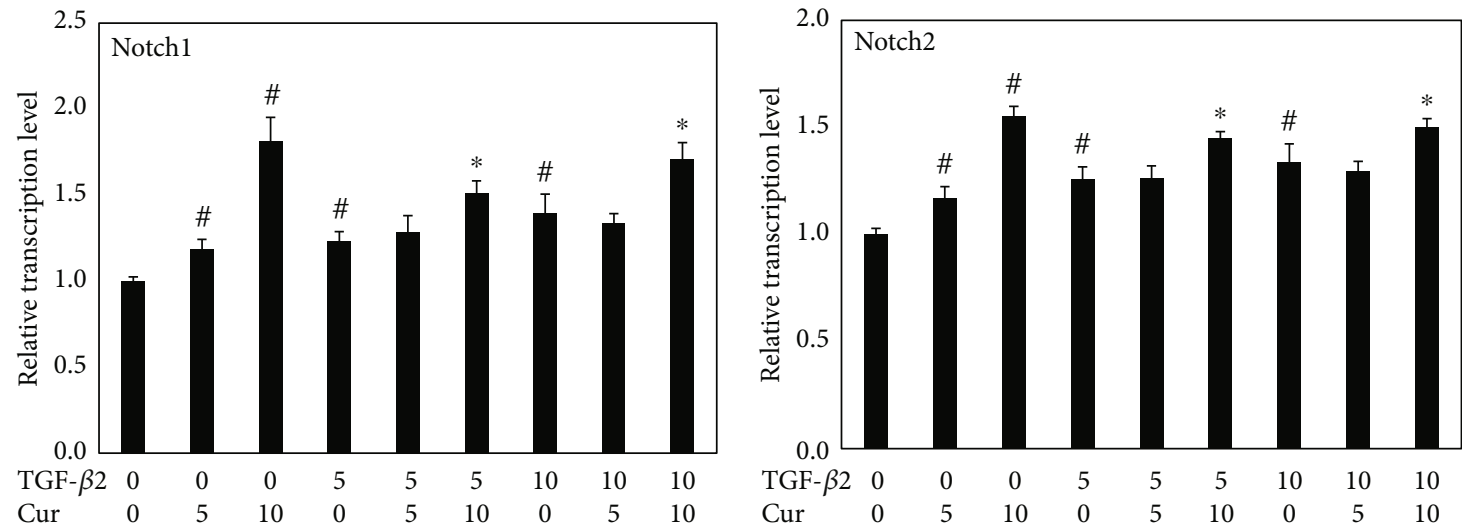

(a)

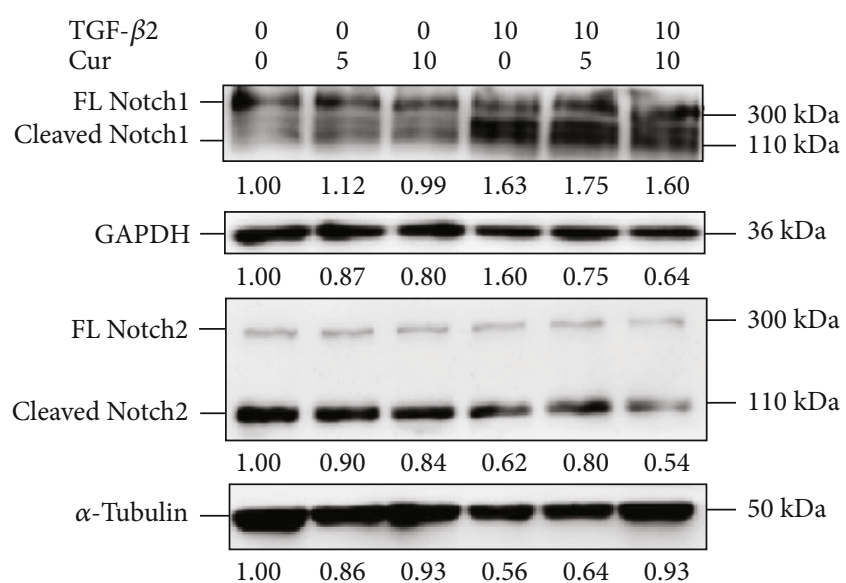

(b)
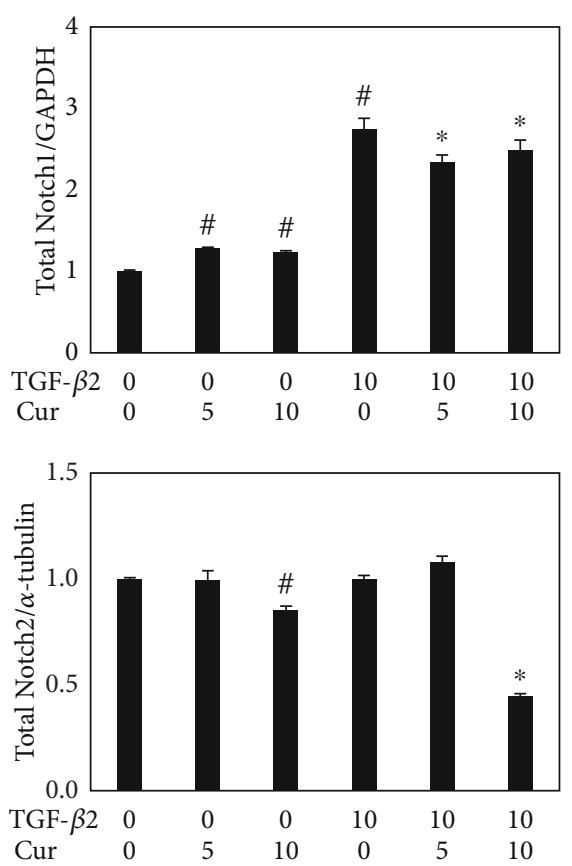

(c)

FIGURE 6: TGF- $\beta 2$ and curcumin activated the transcription of Notch 1 and Notch2, while curcumin decreased the expression of Notch1 and Notch 2 when coincubated with TGF- $\beta 2$ in a dose-dependent way. (a) Normalized mRNA levels of Notch1 and Notch 2 in cells treated with TGF- $\beta 2$ and curcumin for $48 \mathrm{~h}$ were examined by real-time PCR. (b) Protein levels of Notch1 and Notch2 with TGF- $\beta 2$ and curcumin treatments for $48 \mathrm{~h}$ were detected by western blots. (c) Densitometric analyses of the protein expression levels of Notch1 and Notch 2 from the western blots are shown. GAPDH and tubulin were used as internal controls. ${ }^{\#} P<0.05$ vs. the control, ${ }^{*} P<0.05$ vs. treatment with TGF- $\beta 2$ only.

the medical expenses, suffering of patients, and possible risks of complications. From the fact that more patients are receiving extracapsular lens extraction surgery worldwide, PCO has developed into a social burden. Therefore, discovering novel and brief therapeutic methods is urgently required. Curcumin, a component extracted from spice turmeric in the first place, presented abilities of suppressing cellular proliferation and inducing apoptosis in cancers [16, 24, 25]. In this research, we studied the functional mechanism of curcumin on the proliferation and EMT of human LEC cell line SRA01/04 and revealed its valuable potential in solving PCO.

Following the experiments on cellular proliferations, the OD values of LECs were suppressed by curcumin according to a time-dose manner (Figure 1(a)). And at doses under
$10 \mu \mathrm{M}$, curcumin showed no signs of increasing apoptosis (Figures 1(b) and 1(c)), so further studies were performed with curcumin of 0,5 , and $10 \mu \mathrm{M}$. Experiments afterwards had also shown inhibiting effects of curcumin on LEC proliferation. The $G_{2} / M$ phase arrest uncovered the possible time point where proliferating was stopped (Figure 2). In the process of cell cycle [26-29], firstly, cyclin B1 is an important protein required in $\mathrm{G}_{2} / \mathrm{M}$ transition; secondly, phosphorylation of Thr14, Tyr15, and Thr161 in CDK1 by kinases upstream such as Myt1 and WEE activate the CDK1-cyclin $B 1$ complex that regulates phase transition from $G_{2}$ to $M$; thirdly, CDC25C activates the complex through dephosphorylating Thr14/Tyr15 and then initiates the process of mitosis. The downregulation of these cell cycle time point proteins 


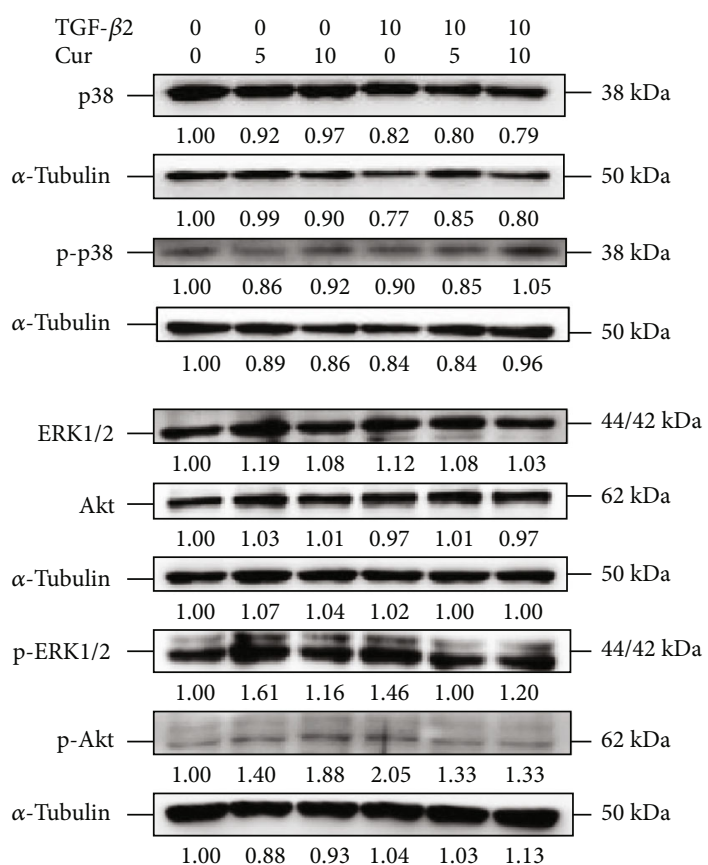

(a)
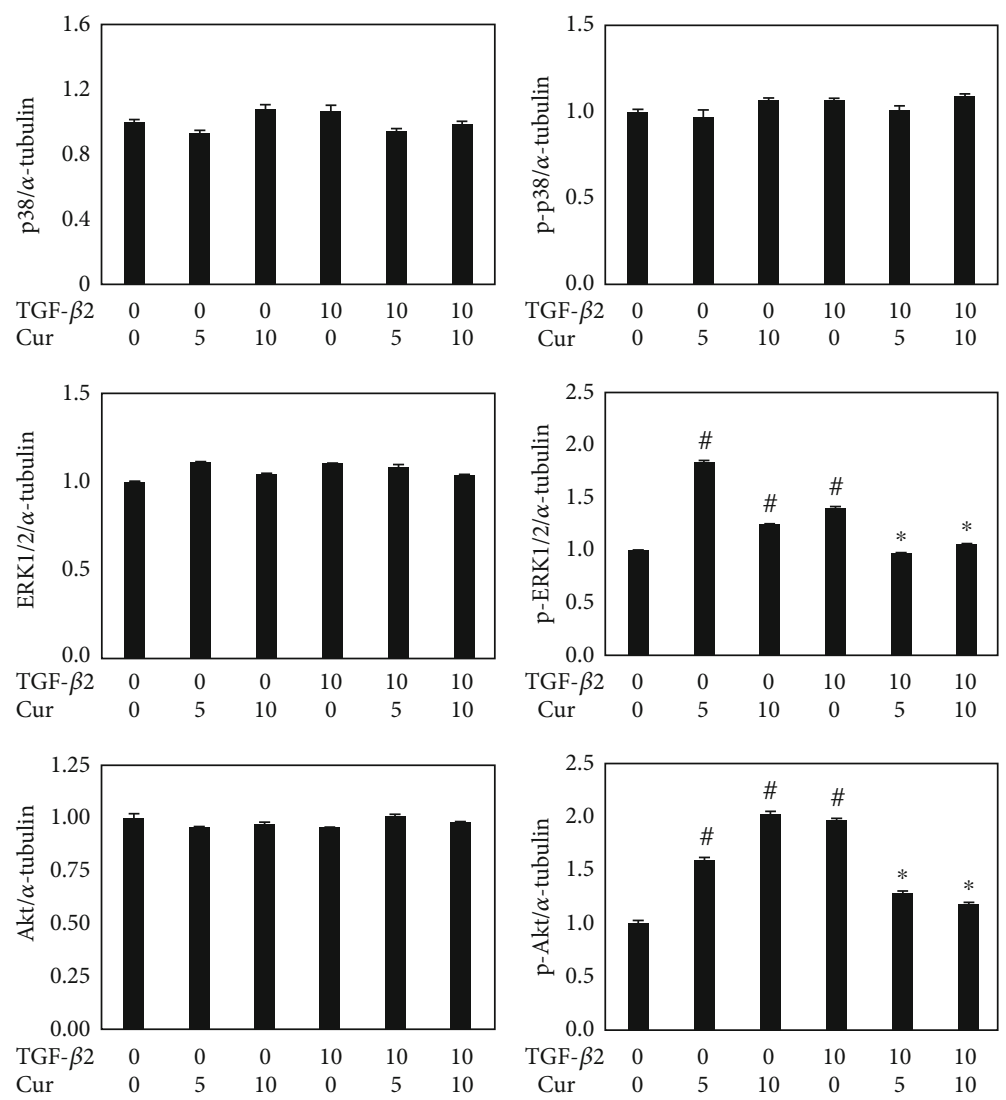

(b)

FIgURE 7: Curcumin suppressed TGF- $\beta 2$-induced activation of the ERK1/2 and Akt pathway while the p38 pathway was not affected. (a) Expressions of p38, ERK1/2, Akt, and their phosphorylated forms in LECs treated with TGF- $\beta 2(0,10 \mathrm{ng} / \mathrm{ml})$ and curcumin $(0,5$, and $10 \mu \mathrm{M}$ ) for $48 \mathrm{~h}$ were detected by western blots. (b) Densitometric analyses of the protein expression levels of p38, p-p38, ERK1/2, p-ERK1/2, Akt, and p-Akt from the western blots are shown. GAPDH and tubulin were used as internal controls. ${ }^{\# P}<0.05$ vs. the control, ${ }^{*} P<0.05$ vs. treatment with TGF- $\beta 2$ only. 
resulted from curcumin treatment explaining the molecular mechanism of $\mathrm{G}_{2} / \mathrm{M}$ phase arrest in LECs (Figure 3). As for curcumin of $5 \mu \mathrm{M}$, it increased the expression of CDK1; it is reported by Chen et al. that a low dose of curcumin $(5 \mu \mathrm{M})$ inhibits proliferation of colorectal cancer cells by inducing senescence rather than apoptosis [30], and besides, Müllers et al. showed the activity of CDK1/2 after DNA damage promotes senescence [31], so the low dose of curcumin may lead to senescence via CDK1 presumably. On the other hand, it was reported that TGF- $\beta 2$ could activate the Akt pathway in human subconjunctival fibroblasts, and Akt along with the MAPK pathway contributed to TGF- $\beta 2$-induced upregulation of Jagged- 1 in retinal epithelial (RPE) cells $[32,33]$. We showed that the mechanism of growth arrest caused by curcumin in LECs under TGF- $\beta$ treatment was related to ERK1/2 blockage and Akt inhibition but not p38 (Figure 7). As all is presented, curcumin is able to inhibit the cellular proliferations of LECs and shows a possible capability in controlling cell growth in PCO.

TGF- $\beta$ is an effective EMT inducer in both normal and pathological conditions [7] and a promoter of mesenchymal transitions in cancer cells [34]. In a previous research, TGF- $\beta$ was also demonstrated to induce EMT in lens, which increased the possibility of PCO onset [1]. In this study, $10 \mathrm{ng} / \mathrm{ml}$ of TGF- $\beta 2$ successfully induced EMT in LECs by elevating protein markers including fibronectin, collagen I, and collagen IV. After treatment of curcumin, the RNA transcriptional levels of the markers fibronectin, collagen $I$, and collagen $I V$ were downregulated (Figure 4). Correspondingly, the protein levels of fibronectin and $\alpha$-SMA were decreased (Figure 5). However, E-cadherin was found to be upregulated by curcumin (Figure 5). This can be explained that curcumin abrogated the mesenchymal feature induced by TGF- $\beta 2$ and restored the epithelial feature of LECs. Research showed that baicalin exerts antifibrosis effects via blocking the TGF- $\beta /$ Smad signaling pathway [35]. Thus, the TGF$\beta /$ Smad signaling pathway is a potential target of alleviating pathogenic-fibrosis processes. In this study, curcumin had downregulated the level of $\mathrm{p}-\mathrm{Smad} 2 / 3$ (Figure 5). It is known that the TGF- $\beta$ signaling pathway is activated by $\operatorname{Smad} 2 / 3$ phosphorylation, causing various processes such as EMT and organ fibrosis [1]. When binding to the receptor, TGF$\beta$ activates the TGF- $\beta /$ Smad signaling pathway and regulates the expression of target genes [36]. Hence, curcumin abrogated EMT by inactivating the $\mathrm{p}$-Smad $2 / 3$ in TGF- $\beta 2$-treated LECs. The Notch pathway was also involved in the process. It was reported that Notch acts as an oncogene and also as a tumor suppressor depending on the context, which is playing an important role in TGF- $\beta 2$-induced EMT $[37,38]$. Further researches had shown that the Jagged/Notch pathway is necessary for EMT induced by TGF- $\beta$ in RPE, while there is a complex interplay existing between ERK1/2, TGF- $\beta / \mathrm{Smad}$, and Jagged/Notch pathways in EMT regulation [4, 33, 39]. Although the transcriptional level and translational level of Notch1 and Notch2 seemed to be in different patterns as shown in the results (Figure 6), it highlighted the possible role of posttranscriptional modulation. And from the protein level, we found that curcumin attenuated the increase of Notch 1 and 2 with TGF- $\beta$ incubation in a dose-dependent manner which may shed light on researches in the future. Put together, the process of EMT in LECs was attenuated by curcumin via various accesses involving TGF- $\beta / \mathrm{Smad}$ and Notch signaling pathways.

Recently, a lot of researches have focused on the mechanism of curcumin's inhibition of malignant tumor cells as well as its function on noncoding RNAs which play an important role in the oncogenesis via the transcriptional and posttranscriptional levels. The main noncoding RNAs in this process include microRNA ( 22 nts) and long noncoding RNA (>200 nts), and they can both act as tumor suppressors or tumor oncogenes in tumorigenesis [40]. Oncogene lncRNA HOTAIR is upregulated in numerous tumors and may be involved in curcumin-induced inhibition of renal carcinoma cell metastasis [41]. In colorectal cancer, curcumin regulates the tumor suppressor GAS5 to modulate the metastatic pathways [42]. It is also showed that curcumin produces repression of miRNA-21 expression, and PTEN the downstream target gene is markedly increased in A549 cells upon incubation with curcumin, finally leading to a marked inhibition of cell growth and inducing apoptosis [43]. Considering noncoding RNAs' involvement in the inhibition of proliferation and metastasis in cancer cells under curcumin incubation, we may speculate that noncoding RNAs could also be regulated by curcumin in the proliferation and EMT of LECs, and further investigation will be needed in the future.

In conclusion, this study has revealed the potential value of curcumin in PCO treatment for the first time. It has not only provided evidence that curcumin could suppress LECs proliferation by $\mathrm{G}_{2} / \mathrm{M}$ cell cycle arrest and blocking ERK1/2 along with Akt pathway but also showed that the curcumin had inhibited TGF- $\beta$-induced EMT through suppressing the TGF- $\beta /$ Smad pathway and interfered with the Notch pathway. Though there are still works to be done to further understand the effects and functional mechanisms of curcumin on cellular pathology, our work has illustrated its possibly curative effect in PCO.

\section{Data Availability}

In this manuscript 6061894 titled "Curcumin Inhibits Proliferation and Epithelial-mesenchymal Transition in Lens Epithelial Cells through Multiple Pathways" submitted to BioMed Research International, the nature of the data is figures in the manuscript. So everyone can access them when reading without any restrictions on data access.

\section{Conflicts of Interest}

The authors declare no competing financial interests.

\section{Acknowledgments}

The LEC cell line SRA01/04 was kindly provided by Professor $\mathrm{Fu}$ Shang at Laboratory Nutrition and Vision Research (Boston, MA, USA), and we are also grateful to Dr. Xiaoyun Chen for her technical guidance. Huijun Liu, Yuxiang Mao and Bing Xia contributed equally. This study was supported by the National Natural Science 
Foundation of China (81670874), the Natural Science Foundation of Guangdong Province, China (2017A030313613), and the Fundamental Research Funds of the State Key Laboratory of Ophthalmology.

\section{References}

[1] F. J. Lovicu, E. H. Shin, and J. W. McAvoy, "Fibrosis in the lens. Sprouty regulation of TGF $\beta$-signaling prevents lens EMT leading to cataract," Experimental Eye Research, vol. 142, pp. 92-101, 2016.

[2] H. Nino, "Prevention of posterior capsule opacification by an intracapsular open capsule device," Investigative Ophthalmology \& Visual Science, vol. 55, no. 7, p. 4014, 2014.

[3] R. H. Trivedi and M. E. Wilson, "Posterior capsule opacification in pediatric eyes with and without traumatic cataract," Journal of Cataract \& Refractive Surgery, vol. 41, no. 7, pp. 1461-1464, 2015.

[4] X. Chen, W. Xiao, W. Chen, L. Luo, S. Ye, and Y. Liu, "The epigenetic modifier trichostatin a, a histone deacetylase inhibitor, suppresses proliferation and epithelial-mesenchymal transition of lens epithelial cells," Cell Death \& Disease, vol. 4, no. 10, article e884, 2013.

[5] H. Lin, H. Ouyang, J. Zhu et al., "Lens regeneration using endogenous stem cells with gain of visual function," Nature, vol. 531, no. 7594, pp. 323-328, 2016.

[6] G. Zhang, L. Kang, J. Chen et al., "CtBP2 regulates TGF $\beta 2$ induced epithelial-mesenchymal transition through Notch signaling pathway in lens epithelial cells," Current Eye Research, vol. 41, no. 8, pp. 1057-1063, 2016.

[7] J. Zavadil and E. P. Bottinger, "TGF-beta and epithelial-tomesenchymal transitions," Oncogene, vol. 24, no. 37, pp. 5764-5774, 2005.

[8] S. Prasad, S. C. Gupta, A. K. Tyagi, and B. B. Aggarwal, "Curcumin, a component of golden spice: from bedside to bench and back," Biotechnology Advances, vol. 32, no. 6, pp. 10531064, 2014.

[9] E. Pecheur, "Curcumin against hepatitis C virus infection: spicing up antiviral therapies with 'nutraceuticals'?" Gut, vol. 63, no. 7, pp. 1035-1037, 2014.

[10] M. James, C. Iwuji, G. Irving et al., "Curcumin inhibits cancer stem cell phenotypes in ex vivo models of colorectal liver metastases, and is clinically safe and tolerable in combination with FOLFOX chemotherapy," Cancer Letters, vol. 364, no. 2, pp. 135-141, 2015.

[11] A. Belkacemi, S. Doggui, L. Dao, and C. Ramassamy, "Challenges associated with curcumin therapy in Alzheimer disease," Expert Reviews in Molecular Medicine, vol. 13, 2011.

[12] B. B. Aggarwal, S. C. Gupta, and B. Sung, "Curcumin: an orally bioavailable blocker of TNF and other pro-inflammatory biomarkers," British Journal of Pharmacology, vol. 169, no. 8, pp. 1672-1692, 2013.

[13] Y. Yang, Y. F. Su, H. W. Yang, Y. H. Lee, J. I. Chou, and K. C. Ueng, "Lipid-lowering effects of curcumin in patients with metabolic syndrome: a randomized, double-blind, placebocontrolled trial," Phytotherapy Research, vol. 28, no. 12, pp. 1770-1777, 2014.

[14] V. Soetikno, K. Suzuki, P. T. Veeraveedu et al., "Molecular understanding of curcumin in diabetic nephropathy," Drug Discovery Today, vol. 18, no. 15-16, pp. 756-763, 2013.
[15] P. Neerati, R. Devde, and A. Gangi, "Evaluation of the effect of curcumin capsules on glyburide therapy in patients with type2 diabetes mellitus," Phytotherapy Research, vol. 28, no. 12, pp. 1796-1800, 2014.

[16] S. Prakobwong, S. C. Gupta, J. H. Kim et al., "Curcumin suppresses proliferation and induces apoptosis in human biliary cancer cells through modulation of multiple cell signaling pathways," Carcinogenesis, vol. 32, no. 9, pp. 1372-1380, 2011.

[17] P. Killian, E. Kronski, K. M. Michalik et al., "Curcumin inhibits prostate cancer metastasis in vivo by targeting the inflammatory cytokines CXCL1 and -2," Carcinogenesis, vol. 33, no. 12, pp. 2507-2519, 2012.

[18] R. Gogada, M. Amadori, H. Zhang et al., "Curcumin induces Apaf-1-dependent, p21-mediated caspase activation and apoptosis," Cell Cycle, vol. 10, no. 23, pp. 4128-4137, 2011.

[19] P. Tveden-Nyborg, T. K. Bergmann, and J. Lykkesfeldt, "Basic \& clinical pharmacology \& toxicology policy for experimental and clinical studies," Basic \& Clinical Pharmacology \& Toxicology, vol. 123, no. 3, pp. 233-235, 2018.

[20] H. Liu, W. Liu, X. Zhou et al., "Protective effect of lutein on ARPE-19 cells upon H2O2-induced G2/M arrest," Molecular Medicine Reports, vol. 16, no. 2, pp. 2069-2074, 2017.

[21] Y. Fan, Z. Huang, C. Long et al., "ID2 protects retinal pigment epithelium cells from oxidative damage through p-ERK1/2/ID2/NRF2," Archives of Biochemistry and Biophysics, vol. 650, pp. 1-13, 2018.

[22] Z. Huang, S. Lin, C. Long et al., "Notch signaling pathway mediates doxorubicin-driven apoptosis in cancers," Cancer Management and Research, vol. 10, pp. 1439-1448, 2018.

[23] X. Zhou, X. Kuang, C. Long et al., "Curcumin inhibits proliferation and epithelial-mesenchymal transition of retinal pigment epithelial cells via multiple pathways," Current Molecular Medicine, vol. 17, no. 4, pp. 312-319, 2017.

[24] M. Starok, P. Preira, M. Vayssade, K. Haupt, L. Salomé, and C. Rossi, "EGFR inhibition by curcumin in cancer cells: a dual mode of action," Biomacromolecules, vol. 16, no. 5, pp. 16341642, 2015.

[25] M. Khan, S. Gahlot, and S. Majumdar, "Oxidative stress induced by curcumin promotes the death of cutaneous T-cell lymphoma (HuT-78) by disrupting the function of several molecular targets," Molecular Cancer Therapeutics, vol. 11, no. 9, pp. 1873-1883, 2012.

[26] J. Wei, H. Su, Y. Bi, J. Li, L. Feng, and W. Sheng, “Anti-proliferative effect of isorhamnetin on HeLa cells through inducing G2/M cell cycle arrest," Experimental and Therapeutic Medicine, vol. 15, no. 4, pp. 3917-3923, 2018.

[27] P. Takac, M. Kello, M. B. Pilatova et al., "New chalcone derivative exhibits antiproliferative potential by inducing G2/M cell cycle arrest, mitochondrial-mediated apoptosis and modulation of MAPK signalling pathway," Chemico-Biological Interactions, vol. 292, pp. 37-49, 2018.

[28] M. Schmidt, A. Rohe, C. Platzer, A. Najjar, F. Erdmann, and W. Sippl, "Regulation of G2/M transition by inhibition of WEE1 and PKMYT1 kinases," Molecules, vol. 22, no. 12, p. $2045,2017$.

[29] H. Harashima, N. Dissmeyer, and A. Schnittger, "Cell cycle control across the eukaryotic kingdom," Trends in Cell Biology, vol. 23, no. 7, pp. 345-356, 2013.

[30] T. Chen, P. Yang, H. Wang, and Z. Y. He, "Silence of long noncoding RNA PANDAR switches low-dose curcumin-induced 
senescence to apoptosis in colorectal cancer cells," OncoTargets and Therapy, vol. 10, pp. 483-491, 2017.

[31] E. Müllers, H. Silva Cascales, K. Burdova, L. Macurek, and A. Lindqvist, "Residual Cdk1/2 activity after DNA damage promotes senescence," Aging Cell, vol. 16, no. 3, pp. 575-584, 2017.

[32] S. Jung, H. K. Lee, J. S. Yoon et al., "Upregulation of TGF-betainduced tissue transglutaminase expression by PI3K-Akt pathway activation in human subconjunctival fibroblasts," Investigative Ophthalmology \& Visual Science, vol. 48, no. 5, pp. 1952-1958, 2007.

[33] X. Chen, W. Xiao, X. Liu et al., "Blockade of jagged/notch pathway abrogates transforming growth factor $\beta 2$-induced epithelial-mesenchymal transition in human retinal pigment epithelium cells," Current Molecular Medicine, vol. 14, no. 4, pp. 523-534, 2014.

[34] B. I. Bassey-Archibong, J. M. Kwiecien, S. B. Milosavljevic et al., "Kaiso depletion attenuates transforming growth factor- $\beta$ signaling and metastatic activity of triple-negative breast cancer cells," Oncogene, vol. 5, no. 3, p. e208, 2016.

[35] Y. Xiao, J. Ye, Y. Zhou et al., "Baicalin inhibits pressure overload-induced cardiac fibrosis through regulating AMPK/TGF- $\beta /$ Smads signaling pathway," Archives of Biochemistry and Biophysics, vol. 640, pp. 37-46, 2018.

[36] B. Park, E. Hwang, S. A. Seo, J. G. Cho, J. E. Yang, and T. H. Yi, "Eucalyptus globulus extract protects against UVB-induced photoaging by enhancing collagen synthesis via regulation of TGF- $\beta$ /Smad signals and attenuation of AP-1," Archives of Biochemistry and Biophysics, vol. 637, pp. 31-39, 2018.

[37] K. Hori, A. Sen, and S. Artavanis-Tsakonas, "Notch signaling at a glance," Journal of Cell Science, vol. 126, Part 10, pp. 2135-2140, 2013.

[38] P. Thacker and D. Karunagaran, "Curcumin and emodin down-regulate TGF- $\beta$ signaling pathway in human cervical cancer cells," PLoS One, vol. 10, no. 3, article e0120045, 2015.

[39] X. Chen, W. Xiao, W. Wang, L. Luo, S. Ye, and Y. Liu, "The complex interplay between ERK1/2, TGF $\beta / \mathrm{Smad}$, and Jagged/Notch signaling pathways in the regulation of epithelial-mesenchymal transition in retinal pigment epithelium cells," PLoS One, vol. 9, no. 5, article e96365, 2014.

[40] Y. Liu, H. Sun, B. Makabel et al., "The targeting of non-coding RNAs by curcumin: facts and hopes for cancer therapy (review)," Oncology Reports, vol. 42, pp. 20-34, 2019.

[41] C. S. Pei, H. Y. Wu, F. T. Fan, Y. Wu, C. S. Shen, and L. Q. Pan, "Influence of curcumin on HOTAIR-mediated migration of human renal cell carcinoma cells," Asian Pacific Journal of Cancer Prevention, vol. 15, no. 10, pp. 4239-4243, 2014.

[42] H. Li, S. Q. Ma, J. Huang, X. P. Chen, and H. H. Zhou, "Roles of long noncoding RNAs in colorectal cancer metastasis," Oncotarget, vol. 8, no. 24, pp. 39859-39876, 2017.

[43] W. Zhang, W. Bai, and W. Zhang, "MiR-21 suppresses the anticancer activities of curcumin by targeting PTEN gene in human non-small cell lung cancer A549 cells," Clinical \& Translational Oncology, vol. 16, no. 8, pp. 708-713, 2014. 